\title{
Perceptual organization of auditory streaming-task relies on neural entrainment of the stimulus-presentation rate: MEG evidence
}

\author{
Ivan Chakalov ${ }^{1,2+}$, Rossitza Draganova ${ }^{2 \dagger}$, Andreas Wollbrink $^{1}$, Hubert Preiss ${ }^{2}$ and Christo Pantev $^{1 *}$
}

\begin{abstract}
Background: Humans are able to extract regularities from complex auditory scenes in order to form perceptually meaningful elements. It has been shown previously that this process depends critically on both the temporal integration of the sensory input over time and the degree of frequency separation between concurrent sound sources. Our goal was to examine the relationship between these two aspects by means of magnetoencephalography (MEG). To achieve this aim, we combined time-frequency analysis on a sensor space level with source analysis. Our paradigm consisted of asymmetric ABA-tone triplets wherein the B-tones were presented temporally closer to the first A-tones, providing different tempi within the same sequence. Participants attended to the slowest B-rhythm whilst the frequency separation between tones was manipulated (0-, 2-, 4- and 10-semitones).

Results: The results revealed that the asymmetric ABA-triplets spontaneously elicited periodic-sustained responses corresponding to the temporal distribution of the A-B and B-A tone intervals in all conditions. Moreover, when attending to the B-tones, the neural representations of the A- and B-streams were both detectable in the scenarios which allow perceptual streaming (2-, 4- and 10-semitones). Alongside this, the steady-state responses tuned to the presentation of the B-tones enhanced significantly with increase of the frequency separation between tones. However, the strength of the B-tones related steady-state responses dominated the strength of the A-tones responses in the 10-semitones condition. Conversely, the representation of the A-tones dominated the B-tones in the cases of 2- and 4-semitones conditions, in which a greater effort was required for completing the task. Additionally, the P1 evoked fields' component following the B-tones increased in magnitude with the increase of inter-tonal frequency difference.

Conclusions: The enhancement of the evoked fields in the source space, along with the B-tones related activity of the time-frequency results, likely reflect the selective enhancement of the attended B-stream. The results also suggested a dissimilar efficiency of the temporal integration of separate streams depending on the degree of frequency separation between the sounds. Overall, the present findings suggest that the neural effects of auditory streaming could be directly captured in the time-frequency spectrum at the sensor-space level.
\end{abstract}

Keywords: MEG, Time-frequency spectrum, Auditory scene analysis, Task-driven entrainment

\footnotetext{
* Correspondence: pantev@uni-muenster.de

${ }^{\dagger}$ Equal contributors

${ }^{1}$ Institute for Biomagnetism and Biosignalanalysis, University of Münster, Malmedyweg 15, 48149 Münster, Germany

Full list of author information is available at the end of the article
} 


\section{Background}

Humans are able to organize perceptually meaningful elements from the mixture of competing sounds in the environment. Albert Bregman has described this phenomenon in the well-known framework of Auditory Scene Analysis [1]. The vast majority of researchers interpret the streaming effect in terms of tonotopic organization of the auditory system [2-17]. According to this interpretation, frequency-distant sounds are processed into distinct neural populations and therefore heard as separate streams, and frequency adjacent sounds are processed in neighboring neural channels leading to their perceptual integration into one unified auditory object. However, it has recently been shown that streams compiled from frequency-remote tones can no longer be heard as distinct sound streams if the tones are presented synchronously rather than successively, despite the enhanced neural activity $[18,19]$. Therefore, the tonotopic organization per se does not explain completely the perception of streaming $[18,19]$. The formation of different auditory streams requires temporal integration of sound input over time [20,21].

On the other hand, numerous studies have evaluated the use of the event-related oscillations to indicate the process of sensory integration in the brain [22-32]. Lins \& Picton, for instance, found that multiple auditory stimuli evoke steady-state activity following their repetition rate [23]. More recent research showed that selective attention could modulate the steady-state responses within the auditory system [33,34] and between different sensory modalities (e.g. between visual and auditory [35,36]). In addition, an electroencephalographic study by Nozaradan and colleagues demonstrated that musical beat could elicit a steady-state response tuned to the beat frequency and, furthermore, that binary and ternary metric interpretation of this beat evoked frequencies tuned to the corresponding imagery meter [37].

With regard to auditory stream segregation, it has been demonstrated that attending to a certain rhythm enhances the magnitude of the steady-state response corresponding to its presentation rate [20]. Nevertheless, these authors investigated the neural representation of two recurring sound sequences separated by a relatively large inter-tonal frequency difference [20] paradigm which primarily produces two auditory streams. An intriguing question, therefore, is how different degrees of inter-tonal frequency separation between the sounds, and respectively different perceptual states, affect the spectral distribution of one and the same polyrhythmic structure.

The present study used magnetoencephalography (MEG) to address this question. Experimental block of three subsequent parts was carried out in which the rhythmic elements were kept constant but the frequency separation between the sounds was systematically manipulated.
Specifically, in the first two parts a variation of the standard ABA-triplet paradigm was used [38], wherein the $\mathrm{B}$-tones were set temporally closer to the first A-tones than to the second A-tones, forming dissimilar rhythms within the same sequence. In the first part, two extreme frequency separations between A- and B-tones, which are respectively known to form the perception of one stream vs. two streams [38], were contrasted (0- vs. 10semitones). In the second part, small (2-semitones) vs. intermediate (4-semitones) frequency separations were opposed, ensuring bi-stable perception [38]. In order to keep sustained attention during all these conditions, the participants were asked to follow the presentation of the $\mathrm{B}$-tones and indicate the switching of their perception from the ABA-rhythm to two separate A- and B-tone streams by pressing a button. A combination of timefrequency analysis on a sensor space level and source analysis were used to analyze the results. We anticipated that in the first part our analysis would capture activity corresponding to the temporal distribution of the A-B and $\mathrm{B}$-A-tone intervals of the ABA-triplets in the singlestream condition (0-semitones). In the streaming condition (10-semitones) presentation frequencies of clear B-tones and A-tones were expected. In the second part (2and 4-semitones) all presentation rates were expected in the spectrum (responses, relevant to presentation rates of both $\mathrm{B}$ - and A-tones and to the asymmetric ABA-triplets). Therefore, the present design provides a complementary model for investigation of stream integration versus stream segregation by varying the inter-tonal frequency separation between the A- and B-tones in a classical streaming task. Accordingly, based on the different presentation rates we could access different perceptual states (integrated versus segregated) by capturing the corresponding frequencies to these different rates in the timefrequency spectrum. Additionally, for the first two parts, source waveforms synchronized to the B-tones of the ABA-structure were extracted, in order to be associated with the time-frequency data.

The third part consisted of a sequence compiled from two independent, simultaneously presented A- and B-tone streams (not as ABA-triplet). In this part the participants were not required to follow any of the rhythms. The A- and B-tones of the two presented sequences appeared always at different temporal relation to each other. Conversely, the temporal distributions between the $\mathrm{A}$ - and the B-tones per se were always regular. The auditory system prefers to organize separate streams based on regularities such as pitch [2] and regular temporal arrangement $[39,40]$. On the other hand, it has recently been demonstrated that streaming can occur without any difference in the fundamental frequency [41] and an integrated percept can occur with irregular arrangements [42]. Therefore, if the auditory system is capable of integrating simultaneously 
the A- and B-tones into separate streams, based on their regular presentation rates and identical tone-frequencies, then two steady-state responses related to their frequency distribution would be captured in the spectrum.

\section{Methods \\ Participants}

Fourteenth right-handed participants (5 males), aged between 22 and 30 years, participated in this study. None of them had a history of otological or neurological disorders. A normal audiological status was verified by puretone audiometry in terms of air conduction hearing thresholds less than $10 \mathrm{~dB}$. Pure-tone thresholds were measured for octave frequency from 250 to $4000 \mathrm{~Hz}$. All participants gave a written, informed consent in accordance with the Declaration of Helsinki. The study protocol was approved by the Ethics Commission of the University of Münster, Germany (WWU-Muenster).

\section{Experimental procedures}

The experiments were organized as experimental block of three following parts. $5 \mathrm{~min}$. silent gaps divided the parts. Non-regular ABA-triplet sequences were used in the first two parts. The stimuli were sinusoidal tone-pips of $25 \mathrm{~ms}$ duration, including $10 \mathrm{~ms}$ rise and decay times. The loudness of the stimuli was set to $60 \mathrm{~dB}$ above the individual hearing thresholds. The duration of each trial was $5 \mathrm{~s}$. The inter trial interval (ITI) was set to $3 \mathrm{~s}$ and the total recording time of one experimental part was 10.6 minutes.

In the first part, the single auditory-stream (object) condition was compared with the streaming-condition ( 0 vs. 10-semitones $\Delta \mathrm{f}$ [frequency difference]), with the exact ordering of these conditions randomized. The second part compared small (2-semitones) versus intermediate (4semitones) $\Delta \mathrm{f}$, in the same way. In all conditions, the frequency of tone A was $500 \mathrm{~Hz}$, but the B-tones were $500 \mathrm{~Hz}$ (single-object condition), $561 \mathrm{~Hz}$ (small $\Delta \mathrm{f}$ ), $630 \mathrm{~Hz}$ (intermediate $\Delta \mathrm{f}$ ) and $891 \mathrm{~Hz}$ for the streaming condition, Figure 1A. In the first two parts, the Sound Onset Asynchrony (SOA) between the successive A-tones was always $250 \mathrm{~ms}$, which corresponds to a presentation rate of $4 \mathrm{~Hz}$. The SOA between the successive B-tones was $500 \mathrm{~ms}$, which corresponds to $2 \mathrm{~Hz}$ presentation rate. The SOA linking the first A-tone and the next B-tone of the asymmetric ABA-structure was $100 \mathrm{~ms}$, thus the SOA between the B-tone and the second A-tone was $150 \mathrm{~ms}$, which corresponds to presentation rates of $10 \mathrm{~Hz}$ and $6.6 \mathrm{~Hz}$, respectively. The SOA between the ABA- triplets was also set to $250 \mathrm{~ms}(4 \mathrm{~Hz})$, Figure 1A. In each experimental part, 80 trials were presented: 40 trials of each condition. The presentation order of the first and second parts was counterbalanced across subjects.

The asymmetric ABA-triplet paradigm used in this study allows two competing perceptual states (integrated vs. segregated). In order to provide an objective estimate of stream integration versus stream segregation we manipulate the $\Delta \mathrm{f}$ between the A- and B-tones whilst keeping sustained attention. Therefore, before the main experiment, the participants were exercised with 10 trials of each condition that allows perceptual streaming (2-, 4- and 10-semitones) from parts 1 and 2 in order to segregate the asymmetric ABA-structure into separate A- and B-streams at the very first moment and to keep the perception as stable as possible. Accordingly, the participants were instructed to focus on the B-tones sequence (in all conditions) and to indicate if their perception switched from the repeated ABA-objects to two segregated $\mathrm{B}$ - and A-tone streams by pressing a mouse button after the presentation of each trial. However, we were not interested in the overall level of performance of this task but in maintaining sustained attention. Moreover, 10 of the present 14 participants took part in our previous research in which all of them were able to hear two streams in case of 2-, 4- and 10-semitones conditions [43]. The 0 -semitones condition is assumed to be always heard as one object.

In the third part, two sequences of independent A- and B-sound-streams (instead of the triplet structure) were presented simultaneously for 5 seconds with an ITI of 3 seconds (80 trials for 15 minutes). The presentation rate of the A-tones, therefore, corresponded to $8 \mathrm{~Hz}$ (SOA = $125 \mathrm{~ms})$ and the B-tones to $4 \mathrm{~Hz}(\mathrm{SOA}=250 \mathrm{~ms})$. The frequency separation was set each time to 10 -semitones (tone $\mathrm{A}=500 \mathrm{~Hz}$;-tone $\mathrm{B}=891 \mathrm{~Hz}$ ), Figure 1B. The subjects were not supposed to pay attention to the stimuli and, instead, watched a silent movie of their choice. However, before the main experiment all of the participants had reported that they can hear two streams in that condition. In additional five-minute part, the spontaneous brain activity was recorded in order to distinguish between the spectral power that corresponds to the expected target frequencies in the third part and the resting brain-state [44]. During the resting state recordings the same experimental design as in part 3 was presented to the participants, however, without audio output to the MEG-room. In that way the same conditions' triggers were available for further epochnig.

\section{MEG data acquisition}

The MEG recording was performed using a 275-channel whole-head system (Omega2005, VSM-Medtech, Port Coquitlam, BC, Canada), sampled at $600 \mathrm{~Hz}$. The participants were seated comfortably in an upright position. The sensors were configured as first-order gradiometers with a baseline of $50 \mathrm{~mm}$. In addition to the MEG, the electrooculogram (EOG) was recorded for subsequent artifact rejection. The participants' head positions were determined at the beginning and at the end of each recording block by 


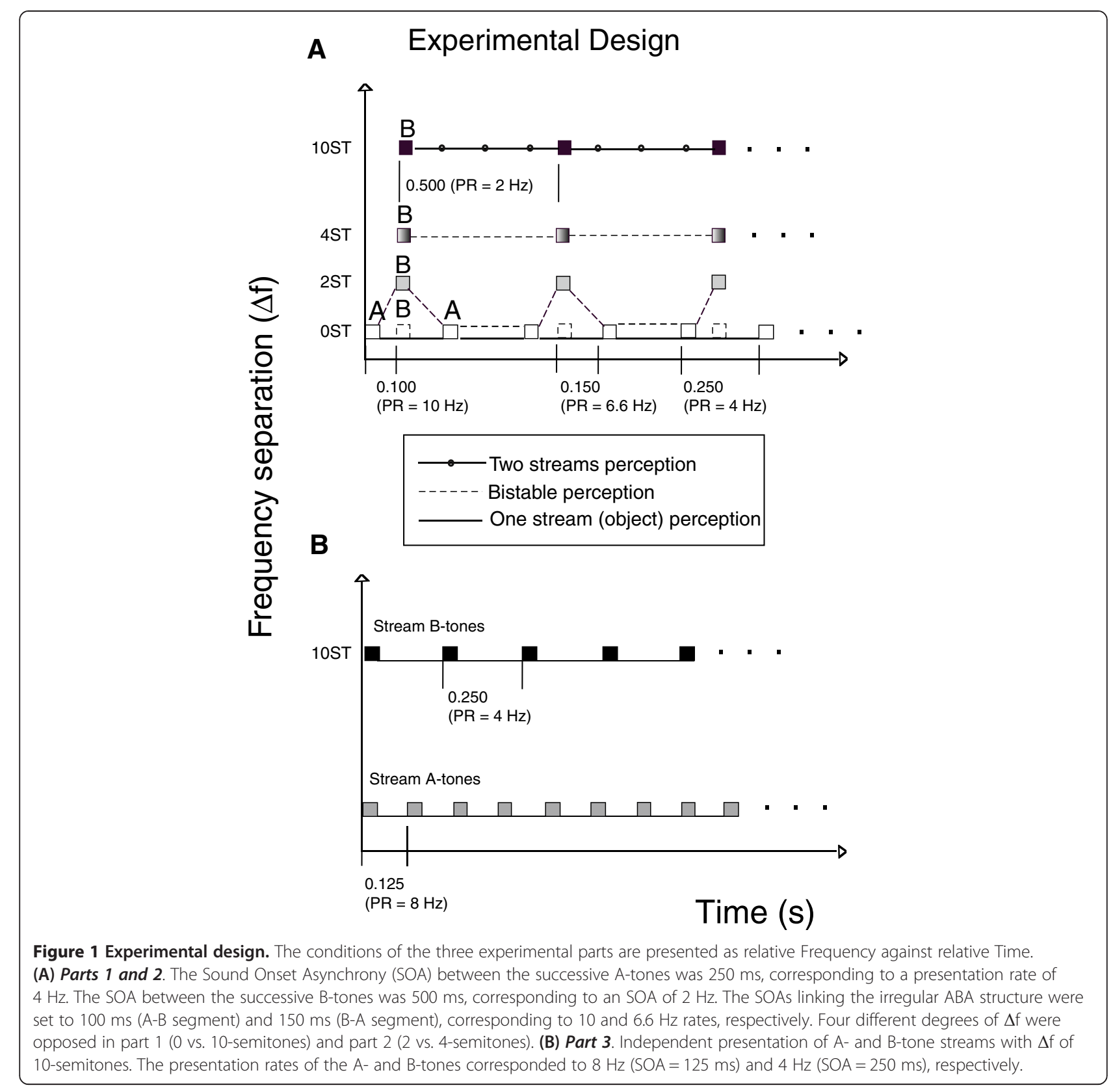

means of 3 localization coils fixed to the nasion and the entrances of both ear canals. Alertness and compliance were verified by video monitoring. The acoustic stimuli were delivered through a nonmagnetic and echo-free acoustic transmission system (VSM-Medtech, Port Coquitlam, BC, Canada) to silicon earpieces placed into the ear canals.

\section{Time-frequency analysis}

In this study we examined whether sequential auditory scene analysis relies on brain oscillations entrained to the stimulus presentation rates. For that purpose we investigated the hypothesized brain frequency oscillations by means of time-frequency analysis. The following MEG processing steps were performed using Matlab-2011a (The MathWorks, Natick, MA, USA) and the FieldTrip toolbox (http://www.ru.nl/neuroimaging/fieldtrip). Before starting the analyses, the continuous data were separated into epochs of $6 \mathrm{~s}$ ( $1 \mathrm{~s}$ before and $5 \mathrm{~s}$ after the onset of the trials). Epochs containing signals larger than $3 \mathrm{pT}$ were considered as artifact-contaminated and excluded from the analysis. In the present study time-frequency representation of power was calculated based on Fourier analysis using a sliding (short time) window approach (mtmconvol). In order to reduce spectral leakage and to control the frequency smoothing prior to power calculation 
the data were multiplied with a single taper (Hanning Window). The length of the sliding window was set to a fixed number of periods resulting in shorter time windows with increasing frequency. To compensate the expense of frequency smoothing for higher frequencies whilst keeping a constant time window (from -1 to $5 \mathrm{~s}$ ) we chose to independently analyze the data for two separate frequency ranges: one from 1 to $6 \mathrm{~Hz}$ and another one from 6 to $14 \mathrm{~Hz}$. Frequency-dependent time window of 2 cycles was used to calculate the activity of the first range (from 1 to $6 \mathrm{~Hz}$ ) and frequency-dependent time window of 13 cycles to calculate the second range (from 6 to $14 \mathrm{~Hz}$ ). For each range the time-frequency power representation was calculated using a frequency resolution of $0.67 \mathrm{~Hz}$ and a temporal resolution of $50 \mathrm{~ms}$.

The topographic maps were also analyzed across the conditions depicting the averaged time course of each stimulus condition and each frequency range (see Additional file 1A), although the goal of the present study was not to focus on concrete brain structures. Based on the prior assumption of different hemispheric specialization in streaming [43] and the obtained dissimilar distribution of the grand averaged topography maps, the magnitudes of the proposed frequency ranges were analyzed separately for the left and right hemispheric channels (cf. Figure 2). The external frontal channels (above the supra-orbital ridges) and the EOG-channels
A OST (one object)10ST (two streams)
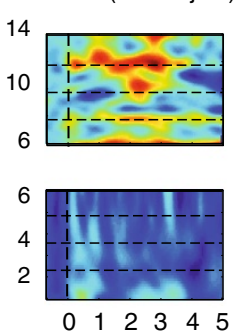

B

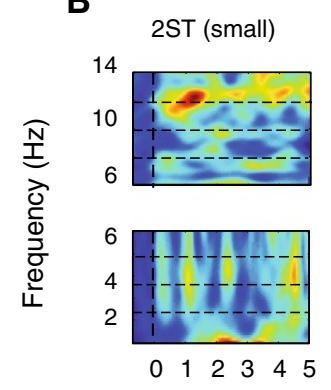

C

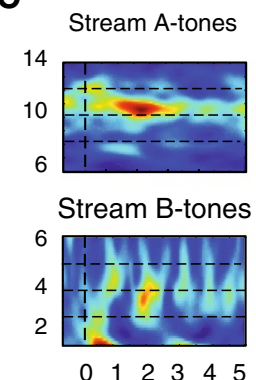

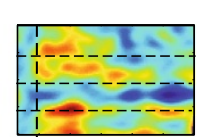

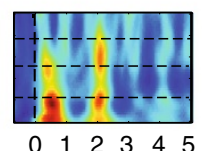

4ST (intermediate)
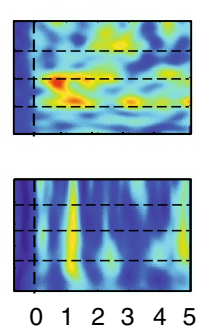

$\begin{array}{llllll}0 & 1 & 2 & 3 & 4 & 5\end{array}$

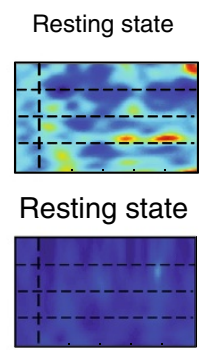

$\begin{array}{llllll}0 & 1 & 2 & 3 & 4 & 5\end{array}$

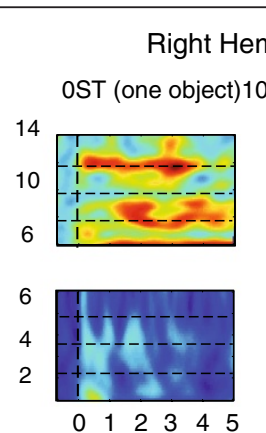

$\begin{array}{llllll}0 & 1 & 2 & 3 & 4 & 5\end{array}$

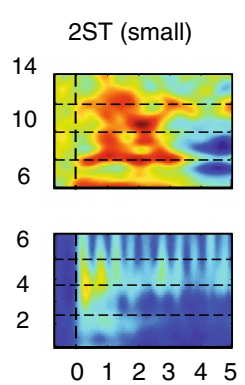

Stream A-tones

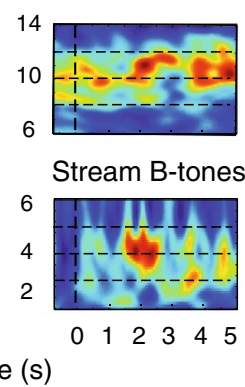

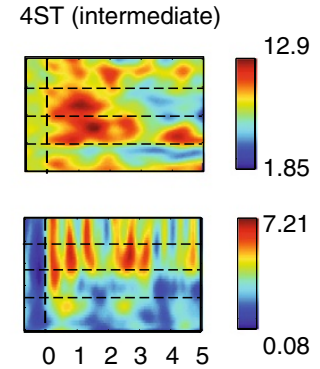

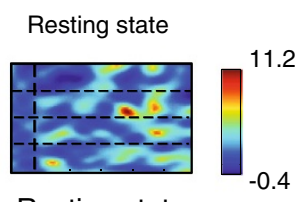

Resting state

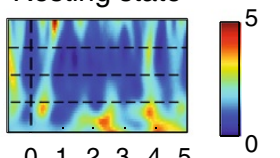

Figure 2 Group level time-frequency plots. The data were averaged across all trials and pre-selected MEG channels separately for the Left (LH) and Right (RH) Hemispheres. (A) Part 1. Non-streaming vs. streaming conditions are presented independently for LH and RH. Upper plots represent the frequency range from 6 to $14 \mathrm{~Hz}$; lower plots the range from 1 to $6 \mathrm{~Hz}$. (B) Part 2. Small vs. intermediate $\Delta$ f conditions are presented in the same way as part 1. (C) Part 3. The plots show simultaneous presentation of independent A- and B-sound streams and the relevant resting brain-state. The two frequency ranges; from 6 to $14 \mathrm{~Hz}$ (upper plots) and from 1 to $6 \mathrm{~Hz}$ (lower plots) are presented separately for the $\mathrm{LH}$ and $\mathrm{RH}$. Color bars represent the relative change of the spectral power. The strength of the signal is color-coded: high strength is denoted with red and low strength with blue. 
were excluded from further analyses in order to additionally diminish the effects of eye blinks and frontal muscle activities. The most occipital channels were less active related to the other channels and therefore excluded from further analyses. At the end, 70 channels of each hemisphere were analyzed. The large number of channels used minimized the side effects of possible individual channel deviation. It should be noted here, that in order to avoid canceling the activities locked to stimulus rate in case of phase difference, the time-frequency representations were calculated for every trial of each individual (40 trials per condition) before averaging the data across the different conditions and participants. Therefore, we expected to capture the activity of approximately $2 \mathrm{~Hz}$ (B-tones presentation rate) and $4 \mathrm{~Hz}$ (A-tones presentation rate) that corresponds to streaming in the cases of the 10-, 4- and 2-semitones conditions (parts 1 and 2) into the first frequency range. In contrast, the activity reflecting the processing of A-B $(10 \mathrm{~Hz})$ and $\mathrm{B}-$ A $(6.6 \mathrm{~Hz})$ time intervals of the unified ABA objects was expected into the second frequency range. The same frequency ranges were analyzed for the third part and the resting state measurements, in order to capture the steadystate activity corresponding to the independent presentation rates of A- and B-tone streams and the relevant spontaneous activity at $8 \mathrm{~Hz}$ and $4 \mathrm{~Hz}$. The time-frequency analyzed epochs were then averaged for each condition ( 40 epochs per condition in part 1 and part 2 and 80 epochs in part 3) across the channels. This was done independently for the left and right hemispheric channels. In order to extract unrelated outstanding noise, such as spontaneous brain activity, baseline-normalization was applied in terms of Relative Change of Spectral Power (RCSP). The RCSP expresses, for each frequency, the relative increase or decrease of the raw power values with respect to the power in the baseline interval. Thus, if $\mathrm{Pa}$ is the spectral power of the post-trigger time-period (from 0 to $5 \mathrm{~s}$ ) and $\mathrm{Pb}$ is the spectral power of the pre-trigger period (from -1 to $0 \mathrm{~s}$ ) the $R S C P$ value is calculated as:

$$
R C S P=(\mathrm{Pa}-\mathrm{Pb}) / \mathrm{Pb}
$$

In addition, the analyzed mean epochs of each condition were averaged across all the participants with the intention of presenting the group-averaged effects. This procedure provided the grand average $R C S P$ values, separately for the two frequency ranges (from 1 to $6 \mathrm{~Hz}$ and from 6 to $14 \mathrm{~Hz}$ ), in the time window from -1 to $5 \mathrm{~s}$, for each condition and hemisphere. It has been shown that the formation of different auditory streams needs a variable amount of time to build-up [16] therefore averaging across the participants could lead to cancelation of some effects based on the dissimilar individual percept over time. Thus, time-frequency responses of one participant were additionally analyzed (Additional file 1B).
Before entering statistical analysis, the mean RCSP values in the time window from 0 to $5 \mathrm{~s}$ post-trigger period were collected for each participant, condition and hemisphere in the following way: (1) for parts 1 and 2, the activity was extracted and averaged between 1.5 and $2.5 \mathrm{~Hz}$ and between 3.5 and $4.5 \mathrm{~Hz}$, looking for the $2 \mathrm{~Hz}$ B-tones and the $4 \mathrm{~Hz} \mathrm{~A}$-tones related steady-state evoked activity; (2) the activity was also averaged between 5.5 and $8 \mathrm{~Hz}$ and between 9 and $11.5 \mathrm{~Hz}$, to identify frequencies of approximately $6.6 \mathrm{~Hz}$ and $10 \mathrm{~Hz}$ corresponding to the distribution of the tone intervals in the asymmetric ABAtriplets; (3) for part 3, the frequency bands between 3.5 and $4.5 \mathrm{~Hz}$ and between 7 and $9.5 \mathrm{~Hz}$ were used, with the expectation of finding $4 \mathrm{~Hz} \mathrm{~B}$-tones and $8 \mathrm{~Hz} \mathrm{~A}$-tones related steady-state evoked activity. The mean RCSP values of the resting state measurements between 3.5 and $4.5 \mathrm{~Hz}$ and between 7 and $9.5 \mathrm{~Hz}$ were also averaged from the additional resting-state part, in order to compare them with the relevant activity derived from part 3 . The calculated mean values of the baseline corrected spectral power for each target frequency were then entered into statistical analysis.

To investigate whether the activity related to the distribution of the ABA-structure depends on the activity related to separate perception of A- and B-tone-streams (parts 1 and 2), the mean spectral power values of the different target frequencies across the different conditions were entered into $4 \times 4$ repeated-measure ANOVA using within-subject factors Target Frequency $(10,6.6,4$ and $2 \mathrm{~Hz}$ ) and Condition (0, 2, 4 and 10-semitones). Thereafter, the mean spectral power of each target frequency from part 1 and part 2 were separately entered into a $2 \times 4$ repeated-measures ANOVAs, using within-subject factors Hemisphere (left, right) and Conditions (0-, 2-, 4- and 10semitones). Thus, we were also able to explore how the spectral power of identical target frequencies changes across conditions, as well as their effects between the hemispheres.

Additionally, the mean values of the baseline corrected spectral power from part 3 were compared with the relevant mean values of spontaneous activity derived from the resting state-part (approx. $8 \mathrm{~Hz}$ and $4 \mathrm{~Hz}$ ). Therefore, a separate $2 \times 4$ model ANOVA was applied here using within-subject factors Hemisphere (left, right) and Activity (spontaneous activity at approx. $8 \mathrm{~Hz}$ and $4 \mathrm{~Hz}$ and evoked activity at approx. $8 \mathrm{~Hz}$ and $4 \mathrm{~Hz}$ ).

When significant, post hoc pairwise comparisons were performed using paired-samples $t$-tests. The alpha level was set at 0.05 and Bonferroni correction was applied in all analyses.

\section{Analysis of source waveform data}

The analysis was performed using the BESA software package (BESA GmbH, Version 5.7.3) and Matlab-2011a 
(The MathWorks, Natick, MA, USA), waveform-toolbox. Before starting the preprocessing procedure, the data were high pass-filtered with the lowest frequency limit of $1 \mathrm{~Hz}$. The data were separated into epochs corresponding to the B-tones of the ABA-triplets, starting $50 \mathrm{~ms}$ before and ending $400 \mathrm{~ms}$ after the B-tone onset. Epochs containing signals larger than $3 \mathrm{pT}$ were considered as artifacts and excluded from further analysis. Before averaging, the response signals were low pass filtered at $30 \mathrm{~Hz}$. Each different condition was then averaged, in order to achieve the best signal-to-noise ratio. These procedures were performed only for the first two parts. The data of part 3 could not be epoched because no baseline could be derived as a result of the independent presentation of A- and B-tone streams.

The signal space projection technique [45] was used for the analysis of the MEG data. The interval used for the ECD fit ( $30 \mathrm{~ms})$ was placed around the local maximum of the N1 component of the AEF. The N1 dipolar sources evoked by B-tones of the ABA-triplets were less variable across conditions compared to the P1, P2 or N2 sources and, thus, provided a better signal-to-noise ratio. Each N1 dipole parameter was represented by the average of all data points (30 ms interval) around the maximum of the Global Field Power of the magnetic field calculated across the respective subsets of channels. Thereafter, the source space projection method was applied to calculate the components of the transient evoked response (P1, N1, P2 and etc.) [45]. For each participant and condition, two ECDs (one in each hemisphere) were determined by their dipole moment, orientation and spatial coordinates (a goodness of fit larger than $90 \%$ was imposed), a technique justified by other authors (e.g. see [29,46-48]).

A time window of $30 \mathrm{~ms}$ was placed around the individual peaks of $\mathrm{P} 1$ and $\mathrm{N} 1$ of the calculated AEFs in order to collect their amplitudes and latencies for further statistical analysis. Two participants in which the expected N1responses could not be fitted into two dipoles were excluded from further analysis hence the responses of eleven participants were entered into statistics. The averaged amplitudes and latencies of $\mathrm{P} 1$ and $\mathrm{N} 1$ components at the $30 \mathrm{~ms}$ interval were then entered into repeated measures ANOVA with the within-subject factors Hemisphere (Left, Right) and Conditions (0-, 2-, 4- and 10-semitones). When significant, post hoc pairwise comparisons were performed using paired-samples $t$-tests. Bonferroni correction was applied for all analyses.

\section{Results}

Time-frequency data

Interactions between the spectral distribution of the $A B A$-structure and $A$ - and $B$-streams

The polyrhythmic structure used in the present study consisted only of two tones (A and B) organized as an asymmetric ABA-triplet. These two tones could form different rhythms, depending on the listener's current perceptual state [49]. This perceptual state is directly influenced by the inter-tonal frequency separation between the A- and B-tones. Therefore, when the perception is integrated as an ABA-stream, one would expect to capture the corresponding presentation frequencies into the spectrum. Conversely, in case of segregation (streaming), one should be able to capture, separately, the temporal distribution of the A- and B-streams in the spectrum. Hence, by varying the inter-tonal frequency separations, we expected to access integrated versus segregated percepts in the time-frequency spectrum.

As shown at the group data-plots (Figure 2AB), the presentation rate of the B-tones of the ABA-triplet sequences elicited a clear increase of the spectral power at about $2 \mathrm{~Hz}$ in the streaming (10-semitones) and in the intermediate frequency separation (4-semitones) conditions from the first and second parts. Additionally, the A-tones presentation elicited a steady-state like activity at about $4 \mathrm{~Hz}$ in the intermediate (4-semitones) and small frequency separation (2-semitones) conditions. The asymmetric ABA-objects induced enhanced activity in all four conditions (parts 1 and 2) at approximately $10 \mathrm{~Hz}$ and $6 \mathrm{~Hz}$ (Figure 2AB).

The ANOVAs revealed significant interaction between the spectral distribution of the ABA-objects and those of the separated A- and B-streams on the basis of the different frequency separation between the competing tones (main effect Conditions $[F(3,39)=5.335, p<.001]$, Target Frequency $[F(3,39)=18.550, p<.001]$ and interaction Conditions $\mathrm{x}$ Target Frequency $[F(9,117)=2.217$, $p<.05])$.

Post hoc pairwise comparisons showed that the mean spectral power corresponding to separate perception of A- and B-streams has lower amplitude compared to the activity related to the distribution of the ABA-triplets in the conditions of small inter-tonal frequency separation. In particular, the B-tones related activity $(2 \mathrm{~Hz})$ of the nonstreaming scenario (0-semitones) was significantly lower compared to spectral power of the A-B (approx. $10 \mathrm{~Hz}$ ) and B-A (approx. $6 \mathrm{~Hz}$ ) tone intervals, $t(13)=-3.434$, $p<.001$ and $t(13)=-5.620, p<.001$. The A-tones $(4 \mathrm{~Hz})$ related spectral power of the same condition was also significantly lower compared to the activity related to the distribution of the ABA-triplets (A-B [approx. $10 \mathrm{~Hz}],[t(13)=-4.125, p<.001]$ and B-A [approx. $6 \mathrm{~Hz}],[t(13)=-4.312, p<.001])$. Furthermore, the $2 \mathrm{~Hz}$ spectral power (B-tones) in the small frequencyseparation condition (2-semitones) was significantly lower compared to the spectral power corresponding to A-B (approx. $10 \mathrm{~Hz}),(t(13)=-3.702, p<.05)$ and B-A tones (approx. $6 \mathrm{~Hz}),(t(13)=-8.068, p<.001)$. Regarding the 2-semitones condition, the corresponding 
activity to a separate A-stream perception at approx. $4 \mathrm{~Hz}$ was also significantly lower than the $10 \mathrm{~Hz}$ $(t(13)=-3,320, \quad p<.001)$ and $6.6 \mathrm{~Hz} \quad(t(13)=8,044$, $p<.001)$ spectral power. Similarly, concerning the intermediate frequency separation condition (4-semitones), post hoc comparisons revealed decreased activities corresponding to separate presentation of A-tones (approx. $4 \mathrm{~Hz}$ ) and B-tones (approx. $2 \mathrm{~Hz}$ ) as compared to spectral power of the A-B target frequency (approx. $10 \mathrm{~Hz}$ ), $(t(13)=-2.321, p<.05)$ and $(t(13)=-2.321, p<.05)$, respectively.

In order to understand better the source of significant interactions from the previous $4 \times 4$ ANOVA and to explore the effect between the hemispheres, four additional ANOVAs were conducted wherein the mean spectral power of each target frequency was entered separately into repeated-measures $2 \times 4$ ANOVA using withinsubject factors Hemisphere (left, right) and Conditions (0-, 2-, 4- and 10-semitones).

\section{Activity related to a perception of separate A- and B-streams (parts 1 and 2)}

The time-frequency outcome demonstrated that the $2 \mathrm{~Hz}$ activity (B-tones related) in the 10-semitones condition evolved at about $0.5 \mathrm{~s}$ and reached its maximum at about $0.8 \mathrm{~s}$ and $2 \mathrm{~s}$. This was not so well pronounced in the case of 2 - and 4-semitones and did not occur during 0 -semitones condition (Figure 2AB, Table 1).

Regarding the $2 \mathrm{~Hz}$ activity (B-tones related), the ANOVAs revealed a significant difference between the conditions (main effect Condition $[F(3,39)=10.063, p<.001]$ ). The following Post hoc pairwise comparisons showed that the activity at approx. $2 \mathrm{~Hz}$ (B-tones related rhythm) increased significantly with increasing the inter-tonal frequency separation between A- and B-tones. The $2 \mathrm{~Hz}-$ activity of the 10-semitones condition was significantly greater than in the 0 -semitones condition $(t(13)=-3.169$, $p<.05)$ and the 2-semitones condition $(t(13)=2.937$, $p<.05)$. The mean spectral power at $2 \mathrm{~Hz}$ of the 4semitones condition was also significantly higher compared to the 2-semitones condition $(t(13)=-3.967, p<.05)$ and the 0 -semitones condition $(t(13)=-3.934, p<.05)$. The comparison of the mean spectral power at $2 \mathrm{~Hz}$, between the conditions with close inter-tonal frequency difference, did not reveal significance; 2-semitones vs. 0-semitones $(t(13)=-0.812, p=.431)$ and 10 -semitones vs. 4-semitones $(t(13)=-1.901, p=.080)$.

As seen on the group level time-frequency plots, the $4 \mathrm{~Hz}$-activity (A-tones related) showed greater enhancement in the second part (Figure 2B), wherein the frequency difference between the $\mathrm{A}$ - and B-tones was relatively small (2- and 4-semitones) when compared to the first part. The power of the signal at approximately $4 \mathrm{~Hz}$ was therefore more pronounced and better visible than at $2 \mathrm{~Hz}$ in the second part (Table 1, Figure 2B). The baseline corrected mean spectral power here differed significantly across conditions (main effect Conditions $[F(3,39)=6.115, p<.05])$. Post hoc comparisons revealed that the spectral power at $4 \mathrm{~Hz}$ in case of 4-semitones was significantly greater than in case of 2 -semitones condition $(t(13)=-2.333, p<.05)$, the 10 semitones condition $(t(13)=-2.709, p<.05)$ and the 0 -semitones condition $(t(13)=-2.618, p<.05)$. The 4 $\mathrm{Hz}$-activity was also significantly greater in case of 2semitones compared to $\mathrm{t} 10$-semitones $(t(13)=-2.173$, $p<.05)$.

Figure 3 summarizes the differences of the spectral power related to the separate perception of the A-tones $(4 \mathrm{~Hz})$ and B-tones $(2 \mathrm{~Hz})$ of all conditions $(0-, 2-$, 4- and

Table 1 Mean values of the baseline corrected spectral power across the conditions

\begin{tabular}{|c|c|c|c|c|c|c|c|}
\hline \multirow{2}{*}{$\begin{array}{l}\text { Frequency } \\
\mathrm{RH}^{\mathrm{a}}\end{array}$} & \multicolumn{2}{|l|}{ Part-1 } & \multicolumn{2}{|l|}{ Part-2 } & \multicolumn{3}{|l|}{ Part-3 } \\
\hline & $0 \mathrm{ST}$ & $10 \mathrm{ST}$ & $2 \mathrm{ST}$ & $4 \mathrm{ST}$ & A-stream & B stream & $S A^{c}$ \\
\hline $10 \mathrm{~Hz}$ & 3.615 & 5.377 & 12.448 & 12.790 & -- & -- & -- \\
\hline $8 \mathrm{~Hz}$ & -- & -- & -- & -- & 3.653 & -- & -0.334 \\
\hline $6.6 \mathrm{~Hz}$ & 4.021 & 2.236 & 4.785 & 3.447 & -- & -- & -- \\
\hline $4 \mathrm{~Hz}$ & 1.375 & 1.680 & 2.352 & 7.210 & -- & 0.628 & 0.194 \\
\hline $2 \mathrm{~Hz}$ & 0.460 & 2.215 & 1.889 & 5.634 & -- & -- & -- \\
\hline $\mathrm{LH}^{\mathrm{b}}$ & $0 \mathrm{ST}$ & $10 \mathrm{ST}$ & $2 \mathrm{ST}$ & $4 \mathrm{ST}$ & A-stream & B-stream & $S A^{c}$ \\
\hline $10 \mathrm{~Hz}$ & 1.392 & -0.009 & 2.885 & 1.882 & -- & -- & -- \\
\hline $8 \mathrm{~Hz}$ & -- & -- & -- & -- & 4.576 & -- & -0.822 \\
\hline $6.6 \mathrm{~Hz}$ & 3.102 & 0.597 & 2.455 & 1.979 & -- & -- & -- \\
\hline $4 \mathrm{~Hz}$ & 1.636 & 1.568 & 1.850 & 3.439 & -- & 1.475 & 0.416 \\
\hline $2 \mathrm{~Hz}$ & 0.613 & 3.942 & 0.084 & 3.253 & - - & - - & - - \\
\hline
\end{tabular}

${ }^{\mathbf{a}}$ RH-Right Hemisphere; ${ }^{\mathbf{b}}$ LH-Left Hemisphere; 'SA- Baseline Corrected Spontaneous Brain Activity. 


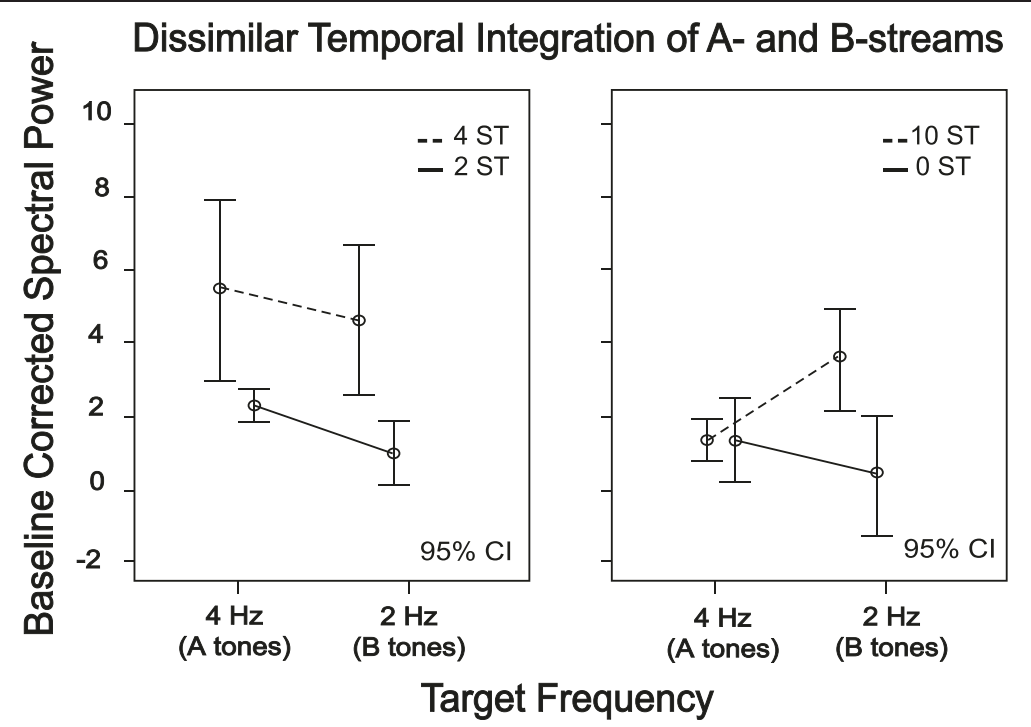

Figure 3 Dissimilar efficiency of the temporal integration of segregated A (approx. $4 \mathrm{~Hz}$ ) and B (approx. $2 \mathrm{~Hz}$ ) streams. The conditions from part 1 (0-vs. 10-semitones) and part 2 (2-vs 4-semitones) are presented on the right and on the left plot, respectively. Error bars indicate the $95 \%$ confidence intervals for the within-subject effect (Condition x Target Frequency).

10-semitones) across the participants. Error bars indicate the $95 \%$ confidence intervals for the within-subject effect (Condition x Target Frequency) [50].

\section{Activity related to distribution of the tone intervals in the asymmetric ABA-triplets (part 1 and 2)}

The activity corresponding to A-B and B-A intervals of the ABA-triplets (approx. 10 and $6 \mathrm{~Hz}$ ) appeared to be sustained during the 0 -semitones condition and transient in the conditions that allowed perceptual streaming (2-, 4- and 10-semitones), Figure 2AB.

The ANOVAs revealed that the mean values of the baseline corrected spectral power at around $10 \mathrm{~Hz}$ (related to the presentation rate of A-B segment in the ABA-triplet) in the first and second part did not change significantly between the conditions (Condition $[F(3,39)=2.588$, $p=.067])$. The activity between the hemispheres, however, differed significantly (main effect Hemisphere $[F(1,13)=$ 17.030, $p<.001]$ ). Post hoc pairwise comparisons showed that the spectral power at $10 \mathrm{~Hz}$ was generally greater in the Right Hemisphere $(R C S P=3.427)$ than in the Left Hemisphere $(R C S P=1.037),(t(13)=-4.281, p<.001)$.

Regarding the $6.6 \mathrm{~Hz}$ target frequency, the ANOVAs revealed significant main effect Condition $(F(3,39)=2.923$, $p<.05)$. The following Post hoc pairwise comparisons showed that the activity at approximately $6 \mathrm{~Hz}$ (B-A interval of ABA-rhythm) was significantly greater in the case of 2 -semitones compared to 10 -semitones $t(13)=2.474$, $p<.05)$. There were no other significant comparisons in the $8 \mathrm{~Hz}$ target frequency $(0-$ vs. 10 -st. $[t(13)=1.954, p=.073]$, 2 - vs 4 -st. $[t(13)=1.293, p=.219]$, 4- vs. 10-st $[t(13)=$
1.705, $p=.112], 0$ - vs. 4-st. [ $t(13)=.884, p=.178]$ and 0 - vs. 2-st $[t(13)=-.156, p=.878])$.

\section{Activity related to the independent presentation of $A$ - and $B$-tones (part 3)}

Unlike the ABA-structure from the first two parts this scenario could not provide two alternative perceptual states (integrated vs. segregated). Therefore, the participants were not required to pay attention to the ongoing presentation. The auditory system prefers regular arrangements $[39,40]$ and hence two steady-state responses corresponding to presentation rates of the two sequences were expected in the time-frequency spectrum. As shown at the plots (Figure 2C), the nonattended condition (part 3) elicited an activity enhancement at about $8 \mathrm{~Hz}$ and $4 \mathrm{~Hz}$, corresponding to the independent $\mathrm{A}$ - and $\mathrm{B}$-tones presentation rates. The baseline corrected mean spectral power values of the third part vs. the Spontaneous activity at $8 \mathrm{~Hz}$ and $4 \mathrm{~Hz}$, across hemispheres, are shown in Table 1.

The ANOVAs showed a significant difference between the different activities (main effect Activity $[F(3,39)=12.759$, $p<.001]$ ). Post hoc pairwise comparisons revealed that the spectral power corresponding to an independent presentation of A- and B-streams (approx. $8 \mathrm{~Hz}$ and $4 \mathrm{~Hz}, \mathrm{RCSP}=$ 9.595) during the stimulation was significantly greater than the spectral power at $8 \mathrm{~Hz}$ and $4 \mathrm{~Hz}$ during the resting state measures $(R C S P=-.852),(t(13)=5.207, p<.001)$.

\section{Source waveform data}

Clearly identifiable evoked responses were obtained from all subjects. The magnitude of the P1 component of the 
responses to $\mathrm{B}$-tones of the $\mathrm{ABA}$-triplets raised with increasing the frequency separation (significant main effect of Condition $[F(3,33)=7.386, p<.001])$, Figure $4 \mathrm{AB}$. Post hoc comparisons revealed that the magnitude of the $\mathrm{P} 1$ component across the trials was significantly greater in the 10-semitones condition than in the 0-semitones $(t(11)=-3.387, \quad p<.05)$ and 4-semitones conditions $(t(11)=2.474, p<.05)$. Additionally, the amplitude was significantly greater in the case of 4-semitones than in the 2 -semitones $(t(11)=-3.101, p<.05)$ and 0 -semitones $(t(11)=-3.720, p<.05)$. There were no other significant comparisons regarding the P1 amplitude (0- vs. 2-st. $[t(11)=1.966, p=.075]$ and 2 - vs 10 -st. $[t(11)=0.457$, $p=.656])$.

The N1 component also increased following the increased frequency separation, however not significantly (Condition $[F(1,11)=1.435, p=.250]$ ). The two components did not show any effects or interactions concerning the hemispheres: P1-effect Hemisphere $(F(3,33)=.164, p=.694)$ and N1-effect Hemisphere $(F(1,11)=.111, p=.746)$. Despite the fact that P2 and N2 were not entered into statistical analyses, it should be noted that they appeared to be enhanced in the case of streaming and intermediate $\Delta \mathrm{f}$, compared to singlestream and small with the lowest frequency limit of $1 \mathrm{~Hz} . \Delta \mathrm{f}$ conditions, respectively (Figure 4AB). These components likely represent the activity related to the second A-tone of the ABA-triplet.

\section{Discussion}

The present study combines time-frequency analysis on a sensor space level with source waveform analysis by means of magnetoencephalography (MEG) to explore the underlying neural activity behind the processing of an ABA-triplet streaming-task. We furthermore challenge the perception by contrasting four degrees of inter-tonal frequency separation and thus enabling the formation of different perceptual states in one and the same polyrhythmic structure. In order to keep sustained attention, the participants were instructed to focus on the slowest rhythm (B-tones). The results of the first two parts (presentation of asymmetric ABA-triplet sequences) revealed a clear increase of the spectral power at approximately $2 \mathrm{~Hz}$ that corresponds to the B-tones presentation rate in the streaming (10-semitones) and intermediate frequency separation (4-semitones)
A

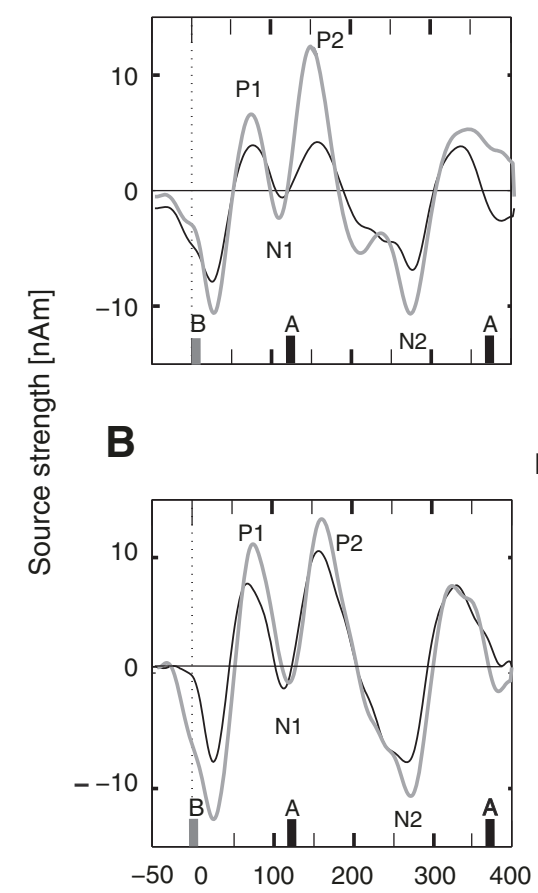

Right Hemisphere

Part 1

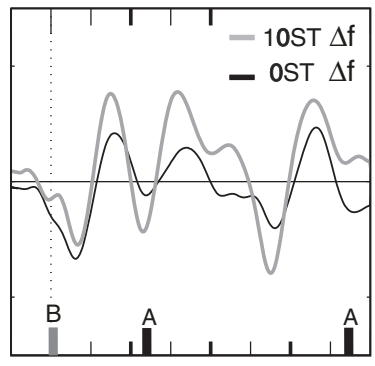

Part 2

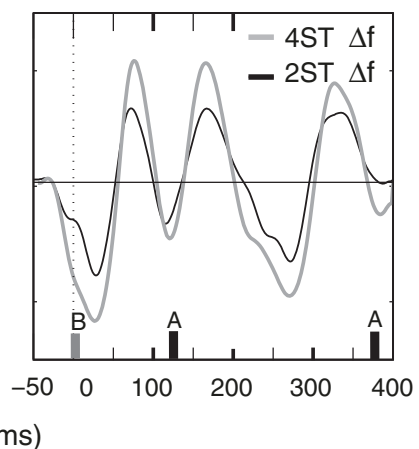

Figure 4 Grand averaged source-waveforms triggered to B-tones of the asymmetric ABA-triplets. The data-from Left and Right Hemispheres are plotted as Source Strength (nAm) against Time (ms). (A) Two different degrees of frequency separation between A- and B-tones $(\Delta f=0$-semitones [black lines] and $\Delta f=10$-semitones [grey lines]) which were presented in the first experimental part are shown. The 0-semitone condition refers to the non-streaming condition and 10-semitones to the streaming condition. (B) The second experimental part presented stimuli of $\Delta f=2$ (black lines) and $\Delta f=4$-semitones (grey lines), referring to small and intermediate $\Delta f$-conditions. 
conditions. This was in line with our hypothesis. Additionally, the A-tones presentation rate elicited steady-state like activity at approximately $4 \mathrm{~Hz}$. The ABA-triplet sequence used in the present study is usually heard as a galloping rhythm and the A- and Bstreams are enclosed into the ABA-pattern [38]. Hence the A- and B-tones related activities at $4 \mathrm{~Hz}$ and $2 \mathrm{~Hz}$ are only accessible in the spectrum if the two streams are segregated. Our results, therefore, likely reflect the selective segregation of the polyrhythmic ABA-pattern into two monorhythmic A- and B- streams. The activity at approximately $10 \mathrm{~Hz}$ and $6 \mathrm{~Hz}$ that corresponds to A$\mathrm{B}$ and $\mathrm{B}$-A-tone intervals of the ABA-triplets also increased across the trials in the first two parts. In the light of the present findings, one might speculate that the neural representation of different auditory sequences relies on neural entrainment of the temporal intervals between the composed stimuli. Therefore, when the perception is in favor of one-stream condition (0-semitones) one could capture the corresponding presentation rates in the spectrum $(10 \mathrm{~Hz}$ and $6.6 \mathrm{~Hz})$, whereas the other rhythms would be suppressed ( $2 \mathrm{~Hz}$ and $4 \mathrm{~Hz}$ ) and vice versa in the case of segregation (10-semitones). Additionally, the time-frequency results demonstrated that the responses to the ABA-frequency distribution (approx.10 Hz and $6.6 \mathrm{~Hz}$ ) appeared to be sustained across the entire presentation of the non-streaming condition (0-semitones), whereas the B-tone related activity $(2 \mathrm{~Hz})$ emerged at approximately $0.5 \mathrm{~s}$ and reached its maxima at approx. $0.8 \mathrm{~s}$ and $2 \mathrm{~s}$ only during the streaming condition (10-semitones). Conversely, the spectral power at $10 \mathrm{~Hz}$ and $6.6 \mathrm{~Hz}$ was rather transient in all other conditions that allowed perceptual streaming (2-, 4- and 10-semitones). The streaming phenomenon is $\mathrm{cu}-$ mulative [51] and needs variable amount of time to build-up [16] and therefore the appearance of the $2 \mathrm{~Hz}$ activity at about $0.5 \mathrm{~s}$ in the time-frequency plots likely reflects the streaming built-up period. Alongside this, the vanishing of the activity at approx. $10 \mathrm{~Hz}$ and $6 \mathrm{~Hz}$ could match the periods wherein the perception alternated in favor of stream segregation. Indeed, the statistical analysis revealed that the spectral power corresponding to the A-B and B-A time intervals of the ABAtriplets is significantly enhanced compared to the responses tuned to the separated $\mathrm{A}$ - and $\mathrm{B}$-tones in the non-streaming scenario (0-semitones) and the conditions of small and intermediate inter-tonal frequency separations (2- and 4-semitones).

The statistical analysis showed furthermore that the steady-state activity related to the attended B-stream $(2 \mathrm{~Hz})$ increased significantly with enlarging the intertonal frequency difference between A- and B-tones (from 0 - to 10-semitones). This result lends further support to the idea that attention is a crucial factor in auditory streaming because it biases the auditory system towards particular grouping or binding of sound-source elements in favor of the listener's intention [19,21]. A previous study by Xiang and colleagues, for instance, explored the mechanisms of temporal integration and its interaction with attention in the auditory system by using a streaming paradigm with two competing tones [20]. The authors demonstrated that focusing the listeners' attention on one of the two competing tempi enhances significantly its steady-state power. However, the two competing tones they used could primarily produce two auditory streams [20], unlike the asymmetric ABAtriplets used in the present study. Furthermore, it has been demonstrated previously that the steady-state responses could be modulated by attention [35,36]. Our experimental design, therefore, allowed us to explore the interaction between the temporal rates in one integrated polyrhythmic pattern and two segregated monorhythmic streams in one and the same tone-sequence. On the other hand, our results revealed a higher spectral power tuned to the A-tones presentation rate $(4 \mathrm{~Hz})$ in comparison with the B-tones related responses $(2 \mathrm{~Hz})$ in the cases of intermediate and small frequency separation between tones, although the attention was focused on the B-rhythm. It might be suggested that in cases of small frequency differences between tones, such as those used in the second part (2- and 4-semitones), the perception of the B-tone is not able to dominate the perception of the A-tones, and that this produces considerably higher activity at approximately $4 \mathrm{~Hz}$ target frequency. It could be speculated therefore, that a greater effort is needed to segregate the ABA-structure onto separate A- and Btone streams in the cases of small and intermediate frequency differences than in the pure streaming condition (10-semitones). In addition, it might be more difficult to follow the slower B-stream $(2 \mathrm{~Hz})$ instead of the twice as fast A-stream $(4 \mathrm{~Hz})$ in the cases of intermediate and small frequency separations than in the greater frequency differences. Besides that, previous studies showed that the steady-state responses are stronger in low frequency rates (below $16 \mathrm{~Hz}$ ) when mediated by attention [21,52]. Although the attention was focused on the B-tones in our experiment, changing the inter-tonal frequency separation into the ABA-tone pattern revealed dissimilar efficiency of temporal integration of separate A- and B-streams. It has been demonstrated previously that the P1 and N1 components of the human AEFs are larger when listeners perceive two segregated streams than one integrated stream and this magnitude augmentation is consistent with the increasing frequency separation between the $\mathrm{A}$ - and B-tones [2]. However, these authors showed that the B-tones' related responses were always enhanced, regardless of the attended stream (A- or B-tones) [2]. Similarly, it has been proposed that the frequency separation between 
different sound sources of a polyrhythmic sequence is sufficient to provide the selective processing of a particular musical instrument; however, the selective attention to one or another spatially separated element of this rhythm could additionally improve the segregation process [53]. These findings together support the idea that the attention in auditory streaming is not merely an intrinsic mechanism that augments the neural responses but its effects are based on a specific interaction between the physical attributes of the stimuli [20]. Additionally, the present outcome is in line with the hypothesis that distinct neuronal populations are involved in the processing of A- and Btones and suppression of one population might underlie the stream segregation phenomenon $[11,12]$.

Assuming that the steady-state activity at low frequency bands is generated by the periodic appearance of the evoked components in response to the A- and B-tones, we tested whether the source waveform of the response signal triggered by the attended B-tones of the ABA-triplets represents any significant effects regarding the evoked peaks. Moreover, the modulation of the source waveforms' components synchronized to each triplet of the ABAstreaming task is a traditional way to investigate the auditory streaming phenomenon (see e.g. [2]). The analysis revealed higher amplitude of the evoked components with increasing the frequency separation, a finding that is in line with prior studies $[2,6,8,54-56]$. Specifically, the P1 evoked component to the B-tones enhanced significantly as the inter-tonal frequency difference increased. This implies that the enhancement of the evoked fields in the source space level together with the B-tones related activity derived from the time-frequency results likely reflect the selective segregation of the attended B-stream. However, the source-wave forms comprise more than one harmonics in the spectrum and it is thus difficult to separate the streaming-related effects from the activities related to the physical features of the sounds. Elhilali and colleagues, for instance, demonstrated that frequency-distant spectral components are no longer heard as separate streams if presented synchronously rather than consecutively, while the neural activity increases with increasing frequency separation between tones [18].

Hence, the auditory evoked fields per se are not capable of fully explaining the perception of streaming.

In apparent contrast to the first two parts, two recurring A- and B-tone-streams were presented in the third part. Here, the temporal distribution between the A-B and BA-tones was always different, whereas the presentation rates of the A-tones and the $\mathrm{B}$-tones per se, were always regular, corresponding to $8 \mathrm{~Hz}$ and $4 \mathrm{~Hz}$, respectively. The results demonstrated clear non-attentive steady-state activity at approx. $8 \mathrm{~Hz}$ and $4 \mathrm{~Hz}$. Indeed, it has been shown that the auditory system prefers regular arrangements $[39,40]$. Moreover, the integration of auditory streams, based on their regularities, could take place automatically. The mismatch negativity component (MMN) of eventrelated potentials, for instance, automatically detects changes in the regular stimulus pattern [57-60]. Additionally, it has been found that the MMN operates also on the basis of auditory objects and that the integration of objects occurs pre-attentively in the auditory system [61]. The experimental design applied in the third part could not provide two complementary percepts (integrated vs. segregated), such as ABA-triplets. It could be speculated, therefore, that the two auditory streams were formed of the very first moment of their presentation. On the other hand, it has recently been demonstrated that streamintegration can occur with irregular arrangements [42]; however, it is likely that in the absence of active awareness, the auditory system integrates tone patterns based on their physical regularities.

In summary, the present findings suggest that neural encoding of a streaming task relies on an oscillatory entrainment of the stimulus presentation rates. However, two separate effects of the time-frequency data must be distinguished: the first is represented in our results by the distribution of the intervals between the A-B $(10 \mathrm{~Hz})$ and $\mathrm{B}-\mathrm{A}(6.6 \mathrm{~Hz})$ tones of the ABA-triplets $(0-$ semitones). The second effect is represented by the $2 \mathrm{~Hz}$ and $4 \mathrm{~Hz}$ steady-state responses related to the B- and Atones derived from the conditions that allow perceptual streaming (2-, 4- and 10-semitones), alongside the steady-state effects of non-attentive listening (part 3). The present effects cannot be directly ascribed to the underlying mechanisms responsible for various perceptual states, because the participants were not required to make streaming judgments during the trials. Nevertheless, these effects might be grounded to physiological hallmarks of the process, which precedes the formation of one vs. two streams percept. Hence, further study is necessary to show the differences in the spectral distribution of identical tonal-frequency separation in conditions of perceptual validation during integration vs. segregation.

\section{Conclusions}

The present findings are consistent with previous studies suggesting that the perceptual organization in sequential auditory scene analysis relies on oscillatory entrainment to task-driven sound input. The results showed that increasing of the frequency separation between A- and Btones of the ABA-pattern correlates with a greater magnitude of the steady-state responses tuned to the attended B-tones. Alongside this, the P1 evoked fields' component, synchronized to the B-tones, increased in amplitude with raising the inter-tonal frequency difference. Furthermore, the asymmetric ABA-objects, spontaneously elicited sustained activity, which corresponded 
to the temporal distribution of the constituent tone intervals. The results also revealed that the efficiency of temporal integration of separate streams is dissimilar depending on the degree of frequency separation between the competing sounds. The steady-state responses tuned to the B-tones dominated the responses tuned to the Atones in the case of great frequency difference between tones. Conversely, the representation of the A-tones dominated the B-tones in the cases of small and intermediate frequency separation, in which the task required greater effort. Overall, the present outcome suggests that the neural effects of auditory stream integration and segregation could be directly captured in the timefrequency spectrum and measured with significance tests at the sensor space level.

\section{Additional file}

\section{Additional file 1: Two separated plots show the Grand Averaged topographic Maps $(A)$ and the neural responses of one Representative Subject (B).}

\section{Competing interests}

The authors declare that they have no financial or any other competing interests.

\section{Authors' contributions}

IC, RD and CP conceived of the study designed the experimental setup and the auditory stimuli. IC acquired the data. IC performed the data \& statistical analyses. All authors participated in the data evaluation and interpretation and in writing the manuscript, and have approved the final version of the manuscript.

\section{Acknowledgements}

We are grateful to Janning Herman for preparation of the Time Frequency Analyses to Evangelos Paraskevopoulos for the helpful comments and Karin Berning for technical assistance. We are very grateful to Ross Parfitt for proofreading the grammar and wording of the paper.

\section{Funding}

This work has been supported by "Deutsche Forschungsgemeinschaft", (DR 807/1-1). The funders had no role in study design, data collection and analysis, decision to publish, or preparation of the manuscript.

\section{Author details}

${ }^{1}$ Institute for Biomagnetism and Biosignalanalysis, University of Münster, Malmedyweg 15, 48149 Münster, Germany. ${ }^{2}$ MEG-Center,

Eberhard-Karls-University Tübingen, Otfried-Müller-Straße 47, 72076 Tübingen, Germany.

Received: 6 June 2013 Accepted: 9 October 2013

Published: 12 October 2013

\section{References}

1. Bregman AS, Liao C, Levitan R: Auditory grouping based on fundamental frequency and formant peak frequency. Can J Psychol 1990, 44(3):400-413.

2. Gutschalk A, Micheyl C, Melcher JR, Rupp A, Scherg M, Oxenham AJ: Neuromagnetic correlates of streaming in human auditory cortex. J Neurosci 2005, 25(22):5382-5388.

3. Snyder JS, Alain C: Age-related changes in neural activity associated with concurrent vowel segregation. Brain Res Cogn Brain Res 2005, 24(3):492-499.

4. Snyder JS, Alain C, Picton TW: Effects of attention on neuroelectric correlates of auditory stream segregation. J Cogn Neurosci 2006, 18(1):1-13.

5. Snyder JS, Alain C: Sequential auditory scene analysis is preserved in normal aging adults. Cereb Cortex 2007, 17(3):501-512.
6. Snyder JS, Holder WT, Weintraub DM, Carter OL, Alain C: Effects of prior stimulus and prior perception on neural correlates of auditory stream segregation. Psychophysiology 2009, 46(6):1208-1215.

7. Snyder JS, Carter OL, Hannon EE, Alain C: Adaptation reveals multiple levels of representation in auditory stream segregation. J Exp Psychol Hum Percept Perform 2009, 35(4):1232-1244.

8. Snyder JS: Weintraub. Pattern specificity in the effect of prior Deltaf on auditory stream segregation. J Exp Psychol Hum Percept Perform: DM; 2011.

9. Pressnitzer D, Hupe JM: Temporal dynamics of auditory and visual bistability reveal common principles of perceptual organization. Curr Biol 2006, 16(13):1351-1357.

10. Bee MA, Klump GM: Auditory stream segregation in the songbird forebrain: effects of time intervals on responses to interleaved tone sequences. Brain Behav Evol 2005, 66(3):197-214.

11. Fishman YI, Arezzo JC, Steinschneider M: Auditory stream segregation in monkey auditory cortex: effects of frequency separation, presentation rate, and tone duration. J Acoust Soc Am 2004, 116(3):1656-1670.

12. Fishman YI, Reser DH, Arezzo JC, Steinschneider M: Neural correlates of auditory stream segregation in primary auditory cortex of the awake monkey. Hear Res 2001, 151(1-2):167-187.

13. Kanwal JS, Medvedev AV, Micheyl C: Neurodynamics for auditory stream segregation: tracking sounds in the mustached bat's natural environment. Network 2003, 14(3):413-435.

14. Micheyl C, Tian B, Carlyon RP, Rauschecker JP: Perceptual organization of tone sequences in the auditory cortex of awake macaques. Neuron 2005, 48(1):139-148.

15. Micheyl C, Carlyon RP, Gutschalk A, Melcher JR, Oxenham AJ, Rauschecker JP, Tian B, Courtenay Wilson E: The role of auditory cortex in the formation of auditory streams. Hear Res 2007, 229(1-2):116-131.

16. Beauvois MW, Meddis R: Time decay of auditory stream biasing. Percept Psychophys 1997, 59(1):81-86.

17. Beauvois MW, Meddis R: Computer simulation of auditory stream segregation in alternating-tone sequences. J Acoust Soc Am 1996, 99(4 Pt 1):2270-2280.

18. Elhilali M, Ma L, Micheyl C, Oxenham AJ, Shamma SA: Temporal coherence in the perceptual organization and cortical representation of auditory scenes. Neuron 2009, 61(2):317-329.

19. Shamma SA, Elhilali M, Micheyl C: Temporal coherence and attention in auditory scene analysis. Trends Neurosci 2011, 34(3):114-123.

20. Xiang J, Simon J, Elhilali M: Competing streams at the cocktail party: exploring the mechanisms of attention and temporal integration. J Neurosci 2010, 30(36):12084-12093.

21. Elhilali M, Xiang J, Shamma SA, Simon JZ: Interaction between attention and bottom-up saliency mediates the representation of foreground and background in an auditory scene. PLOS Biol 2009, 7(6):e1000129.

22. Galambos R, Makeig S, Talmachoff PJ: A 40-Hz auditory potential recorded from the human scalp. Proc Natl Acad Sci USA 1981, 78(4):2643-2647.

23. Lins OG, Picton TW: Auditory steady-state responses to multiple simultaneous stimuli. Electroencephalogr Clin Neurophysiol 1995, 96(5):420-432.

24. Plourde G: Auditory evoked potentials. Best Pract Res Clin Anaesthesiol 2006, 20(1):129-139.

25. Draganova R, Ross B, Borgmann C, Pantev C: Auditory cortical response patterns to multiple rhythms of AM sound. Ear Hear 2002, 23(3):254-265.

26. Ross B, Herdman AT, Pantev C: Stimulus induced reset of $40-\mathrm{Hz}$ auditory steady-state responses. Neurol Clin Neurophysiol 2004, 2004:21.

27. Ross B, Herdman AT, Pantev C: Right hemispheric laterality of human 40 $\mathrm{Hz}$ auditory steady-state responses. Cereb Cortex 2005, 15(12):2029-2039.

28. Ross B, Borgmann C, Draganova R, Roberts LE, Pantev C: A high-precision magnetoencephalographic study of human auditory steady-state responses to amplitude-modulated tones. J Acoust Soc Am 2000, 108(2):679-691.

29. Pantev C, Roberts LE, Elbert T, Ross B, Wienbruch C: Tonotopic organization of the sources of human auditory steady-state responses. Hear Res 1996, 101(1-2):62-74.

30. Basar E, Basar-Eroglu C, Karakas S, Schurmann M: Gamma, alpha, delta, and theta oscillations govern cognitive processes. Int J Psychophysiol 2001, 39(2-3):241-248

31. Schroeder CE, Lakatos P: Low-frequency neuronal oscillations as instruments of sensory selection. Trends Neurosci 2009, 32(1):9-18.

32. Schroeder CE, Lakatos $P$ : The gamma oscillation: master or slave? Brain Topogr 2009, 22(1):24-26. 
33. Muller N, Schlee W, Hartmann T, Lorenz I, Weisz N: Top-down modulation of the auditory steady-state response in a task-switch paradigm. Front Hum Neurosci 2009, 3:1

34. Bohr A, Bernarding C, Strauss DJ, Corona-Strauss Fl: Effects of auditory selective attention on chirp evoked auditory steady state responses. Conf Proc IEEE Eng Med Biol Soc 2011, 2011:2013-2016.

35. Saupe K, Schroger E, Andersen SK, Muller MM: Neural mechanisms of intermodal sustained selective attention with concurrently presented auditory and visual stimuli. Front Hum Neurosci 2009, 3:58.

36. Saupe K, Widmann A, Bendixen A, Muller MM, Schroger E: Effects of intermodal attention on the auditory steady-state response and the event-related potential. Psychophysiology 2009, 46(2):321-327.

37. Nozaradan S, Peretz I, Missal M, Mouraux A: Tagging the neuronal entrainment to beat and meter. J Neurosci 2011, 31(28):10234-10240

38. Van Noorden L: Temporal cocherence in the perception of tone sequences. Eindhoven: University of Technology; 1975.

39. Rimmele J, Schroger E, Bendixen A: Age-related changes in the use of regular patterns for auditory scene analysis. Hear Res 2012, 289(1-2):98-107.

40. Andreou LV, Kashino M, Chait M: The role of temporal regularity in auditory segregation. Hear Res 2011, 280(1-2):228-235.

41. Moore BC, Gockel HE: Properties of auditory stream formation. Philos Trans R Soc Lond B Biol Sci 2012, 367(1591):919-931.

42. Carl D, Gutschalk A: Role of pattern, regularity, and silent intervals in auditory stream segregation based on inter-aural time differences. Exp Brain Res 2013, 224(4):557-570.

43. Chakalov IK, Draganova R, Wollbrink A, Preissl H, Pantev C: Modulations of neural activity in auditory streaming caused by spectral and temporal alternation in subsequent stimuli: a magnetoencephalographic study. BMC Neurosci 2012, 13(1):72.

44. Gross J, Baillet S, Barnes GR, Henson RN, Hillebrand A, Jensen O, Jerbi K, Litvak V, Maess B, Oostenveld R, Parkkonen L, Taylor JR, van Wassenhove V, Wibral M, Schoffelen JM: Good practice for conducting and reporting MEG research. Neuroimage 2012, 65C:349-363.

45. Tesche CD, Uusitalo MA, Ilmoniemi RJ, Huotilainen M, Kajola M, Salonen O: Signal-space projections of MEG data characterize both distributed and well-localized neuronal sources. Electroencephalogr Clin Neurophysiol 1995 95(3):189-200.

46. Pantev C, Okamoto H, Ross B, Stoll W, Ciurlia-Guy E, Kakigi R, Kubo T: Lateral inhibition and habituation of the human auditory cortex. Eur J Neurosci 2004, 19(8):2337-2344.

47. Pantev C, Bertrand O, Eulitz C, Verkindt C, Hampson S, Schuierer G, Elbert T: Specific tonotopic organizations of different areas of the human auditory cortex revealed by simultaneous magnetic and electric recordings. Electroencephalogr Clin Neurophysiol 1995, 94(1):26-40.

48. Pantev C, Elbert T, Makeig S, Hampson S, Eulitz C, Hoke M: Relationship of transient and steady-state auditory evoked fields. Electroencephalogr Clin Neurophysiol 1993, 88(5):389-396.

49. Denham S, Bendixen A, Mill R, Toth D, Wennekers T, Coath M, Bohm T, Szalardy O, Winkler I: Characterising switching behaviour in perceptua multi-stability. J Neurosci Methods 2012, 210(1):79-92.

50. Loftus GR, Masson MEJ: Using confidence intervals in within-subject designs. Psychon Bull Rev 1994, 1:476-490.

51. Bregman AS: Auditory streaming is cumulative. J Exp Psychol Hum Percept Perform 1978, 4(3):380-387.

52. Wang Y, Ding N, Ahmar N, Xiang J, Poeppel D, Simon JZ: Sensitivity to temporal modulation rate and spectral bandwidth in the human auditory system: MEG evidence. J Neurophysio/ 2012, 107(8):2033-2041.

53. Saupe $K$, Koelsch $S$, Rubsamen R: Spatial selective attention in a complex auditory environment such as polyphonic music. J Acoust Soc Am 2010, 127(1):472-480.

54. Snyder JS, Gregg MK, Weintraub DM, Alain C: Attention, awareness, and the perception of auditory scenes. Front Psychol 2012, 3:15.

55. Snyder JS, Alain C: Toward a neurophysiological theory of auditory stream segregation. Psychol Bull 2007, 133(5):780-799.

56. Gutschalk A, Oxenham AJ, Micheyl C, Wilson EC, Melcher JR: Human cortical activity during streaming without spectral cues suggests a general neural substrate for auditory stream segregation. J Neurosci 2007, 27(48):13074-13081.

57. Sussman E, Steinschneider M: Neurophysiological evidence for context-dependent encoding of sensory input in human auditory cortex. Brain Res 2006, 1075(1):165 174.
58. Sussman E, Winkler I, Huotilainen M, Ritter W, Naatanen R: Top-down effects can modify the initially stimulus-driven auditory organization. Brain Res Cogn Brain Res 2002, 13(3):393-405

59. Sussman E, Ritter W, Vaughan HG Jr: An investigation of the auditory streaming effect using event-related brain potentials. Psychophysiology 1999, 36(1):22-34.

60. Hsiao FJ, Wu ZA, Ho LT, Lin YY: Theta oscillation during auditory change detection: an MEG study. Biol Psychol 2009, 81(1):58-66.

61. Ritter W, Sussman E, Molholm S: Evidence that the mismatch negativity system works on the basis of objects. Neuroreport 2000, 11(1):61-63.

doi:10.1186/1471-2202-14-120

Cite this article as: Chakalov et al:: Perceptual organization of auditory streaming-task relies on neural entrainment of the stimulus-presentation rate: MEG evidence. BMC Neuroscience 2013 14:120.

\section{Submit your next manuscript to BioMed Central and take full advantage of:}

- Convenient online submission

- Thorough peer review

- No space constraints or color figure charges

- Immediate publication on acceptance

- Inclusion in PubMed, CAS, Scopus and Google Scholar

- Research which is freely available for redistribution 